\title{
USO DAS FERRAMENTAS LOGÍSTICAS PARA O PLANEJAMENTO DA GESTÃO DOS RECURSOS HÍDRICOS DA REGIÃO NORDESTE
}

Brenna Dafni Fernandes de Oliveira (IFRN - SGA) brennasilva1984@gmail.com José Carlos Guedes da Silva (IFRN - SGA) jcguedes2307@ gmail.com Lucas Barros de Araújo Dantas (IFRN - SGA) lbarros948@gmail.com Paula Victória Dantas Lopes (IFRN - SGA) pvdlopes15@gmail.com Marcus Vinicius Dantas de Assunção (IFRN - SGA) marcus.assuncao@ifrn.edu.br

\section{Resumo}

A água é um recurso natural com grande valor econômico, social e ambiental. A má distribuição e gestão dos recursos hídricos para a região Nordeste, que por sua vez, possui uma presente desigualdade quanto ao abastecimento de água, podem ser severas. Tendo isso como base, o presente artigo tem como objetivo analisar a atual situação dos reservatórios hidrográficas da região Nordeste sob a ótica da implementação do uso de ferramentas da gestão logística com a finalidade de otimizar o planejamento dos recursos hídricos da região. Foi então realizado um estudo descritivo de ordem qualitativa e quantitativa, por meio de recorte temporal e regional, utilizando dados retirados de revistas, artigos científicos e órgãos federais como a ANA (Agência Nacional das Águas) e o IBGE (Instituto Brasileiro de Geografia e Estatística) usufruindo desses dados para a implementação das ferramentas logísticas. Utilizou-se ferramentas como o giro, a cobertura, o estoque médio e a classificação ABC. Essa pesquisa se fez necessária haja vista que os atuais métodos de planejamento utilizados, embora parcialmente eficazes, não se fazem suficientes para atender integralmente às demandas de consumo, de modo que a crise hídrica do Nordeste está sempre em pauta, requerendo investimentos em estudos e novas abordagens de tratamento para esse quadro além de políticas públicas eficientes.

Palavras-chaves: Água, Ferramentas logísticas, Otimização. 


\section{Introdução}

O fortalecimento das práticas da sociedade de consumo contribui para a crescente utilização da água com a finalidade de atender às demandas do mercado pela produção de bens de consumo em larga escala. No entanto, pode-se aplicar um recurso da administração conhecido como Regra de Pareto ou Princípio 80/20 (1906), que afirma que, para muitos eventos, aproximadamente $80 \%$ dos efeitos vêm de $20 \%$ das causas. Ou seja, nesse caso específico, (aproximadamente) $80 \%$ dos recursos hídricos do planeta em condições de uso se destinam ao uso de (aproximadamente) 20\% da população. Então, enquanto uma pequena parcela da população mundial consome quantidades muito além do necessário, a maioria precisa administrar cuidadosamente uma pequena quantidade.

Da mesma forma, no Brasil o problema não é a falta de recursos hídricos, haja vista que o país é detentor da maior reserva de água doce do mundo, e sim sua distribuição de maneira desigual, pois há localidades pouco povoadas e com muitas reservas e outras com uma relação inversa, a exemplo da região Nordeste, que, de acordo com o IBGE, tem o menor percentual de abastecimento diário de água. Em 2018, somando todos os municípios do Nordeste, cerca de $12 \%$ da população da região não tinha essa disponibilidade diária de água. A região também é reconhecida pelas frequentes secas que assolaram sua população ao longo dos tempos, contribuindo para a consolidação do estereótipo do nordestino como povo que vive em condições de miséria.

Dessa forma, as secas típicas do Nordeste brasileiro estão profundamente enraizadas com a caracterização da região como economicamente "fraca" e corroboram com a crença de que se trata de uma região limitante da economia do país. De fato, a seca nunca esteve associada à riqueza, já que a disponibilidade de água é fundamental para os seres humanos desde o desenvolvimento das tarefas mais básicas e individuais até a produção de bens de consumo em larga escala. Todavia, diversos países ao redor do globo encontram-se em regiões geograficamente pouco favorecidas e conseguem se tornar economias relativamente fortes se adaptando ao meio em termos de infraestrutura e gestão de recursos. Então por que no caso específico desta região do Brasil observa-se tal dificuldade para administrar os recursos de forma homogênea e com foco no crescimento?

Trata-se de uma questão complexa de múltiplas variáveis e que, portanto, requer um investimento em estudos detalhados em diferentes áreas. A respeito disso, tem-se que, além da seca, algumas das grandes dificuldades da Região Nordeste que impactam nos baixos níveis de 
abastecimento de água são as altas taxas de evaporação de água nos reservatórios; a construção de desvios ilegais, que dificultam o controle do consumo e por vezes prejudicam o abastecimento dos principais reservatórios; problemas de infraestrutura, os quais provocam vazamentos que podem levar dias para serem solucionados devido a questões burocráticas lentas; bem como diversos outros fatores que tendem a não receber a devida atenção por parte do governo e da própria população.

Diante do exposto, esta pesquisa tem como objetivo analisar a atual situação dos reservatórios hidrográficos da região Nordeste sob a ótica da implementação do uso de ferramentas da gestão logística com a finalidade de otimizar o planejamento dos recursos hídricos da região.

Levando em conta o que foi explicitado, tem-se que a água é um recurso natural com grande valor econômico, social e ambiental. Ademais, a seca do Nordeste está sempre em pauta, no entanto carece de estudos sobre o tema. Tendo em consideração a natureza do objeto estudado, verifica-se a possibilidade de realizar uma nova abordagem, pautada no estudo da gestão hídrica e simultaneamente da gestão de uma cadeia de suprimentos. Por conseguinte, este trabalho, ao utilizar ferramentas logísticas para colocar em evidência as causas da desigualdade no abastecimento de água no Nordeste em comparação ao resto do Brasil, assume grande importância regional, contribuindo para que se tenha ciência do quadro que o nordeste brasileiro vivência. Por fim, pode servir de apoio para futuros estudos que busquem amenizar os impactos desse quadro, seja conscientizando a população ou incentivando a gestão de recursos hídricos integrada a outras áreas.

\section{Referencial teórico}

\subsection{Gestão integrada de recursos hídricos}

Gestão de recursos hídricos, é definida, em sentido amplo, como a forma pela qual se busca equacionar e resolver as questões da escassez relativa da água. Barth engloba na gestão as seguintes funções: planejamento, administração e regulamentação.

Para regulamentar o acesso aos recursos hídricos, a Agência Nacional de Águas (ANA) e os órgãos gestores estaduais utilizam mecanismos de planejamento e coordenação do uso da água no país. Esses mecanismos compõem a gestão de recursos hídricos. 
Um exemplo é a Política Nacional de Recursos Hídricos (PNRH), instituída pela lei $\mathrm{n}^{\circ}$ 9.433, de 8 de janeiro de 1997, também chamada de Lei das Águas. Esta e outras regras orientam a gestão de recursos hídricos no País e promovem o uso múltiplo das águas brasileiras.

Para promover mudanças nas práticas que são consideradas fundamentais para a melhoria da gestão de recursos hídricos, foi desenvolvido na Conferência Internacional sobre Água e meio Ambiente (1992) o conceito de gestão integrada de recursos hídricos.

A gestão integrada dos recursos hídricos (GIRH) foi definida pela Global Water Partnership (GWP) como "um processo que promove a coordenação de desenvolvimento e gestão de água, terra e recursos relacionados, a fim de maximizar a resultante econômico e o bem-estar social de forma equitativa, sem comprometer a sustentabilidade vital dos ecossistemas".

\subsection{Estoque e indicadores de desempenho}

Quanto aos estoques na logística, Viana (2000) diz que são materiais, mercadorias ou produtos acumulados para utilização em momento posterior oportuno, de modo a permitir o atendimento regular das necessidades dos usuários e a continuidade das atividades da empresa. Tendo em vista a natureza fundamental da água para o desenvolvimento de atividades em praticamente todas as áreas e sua necessidade de ser armazenada para garantir o posterior abastecimento de indivíduos e empresas, a mesma será trabalhada como um estoque, possibilitando, por conseguinte a aplicação de recursos logísticos para seu estudo.

Diante do exposto, para verificar o nível de aproveitamento dos estoques, faz-se uso de indicadores de desempenho. Um importante indicador de desempenho é a rotatividade ou o giro de estoque, uma relação existente entre o consumo anual e o estoque médio do produto, dada pela equação 1:

Rotatividade $=$ consumo médio anual/ estoque médio (Equação 1)

Fogarty e Blackstone (1991) definem o giro de estoque como o número de vezes em que o estoque é renovado durante um determinado período. Para conhecer o número de dias em que o estoque permanece parado durante um determinado intervalo de tempo basta dividir o número de dias do período em estudo pelo giro de estoque. O valor encontrado será a cobertura do estoque, ou seja, o índice utilizado para medir o tempo que o estoque, em um determinado período, consegue atender as demandas futuras sem a necessidade de abastecimento. 


\subsection{Cadeia de suprimento e transporte}

Outrossim, posto o armazenamento da água como uma forma de estoque, pode-se afirmar também que sua distribuição integra uma cadeia de suprimento, pois, de acordo com Lummus e Albert (1997), uma Cadeia de Suprimento (Supply Chain) é uma rede de entidades na qual o material flui. Considerando isso, a intervenção de uma infraestrutura de transporte eficiente é de fundamental importância para uma boa gestão hídrica pois realiza o papel de elo entre todas as etapas envolvidas no abastecimento da água.

\subsection{Legislação}

Quanto à gestão de recursos hídricos no Nordeste, os estudos iniciaram-se em 1993, com José Nilson Beserra Campos e Vicente de P.P.B. Vieira, no contexto de análise do cenário institucional e jurídico do Brasil em relação à o estabelecimento de um sistema de gestão de recursos hídricos para o Nordeste. Eles apontam que a base jurídica brasileira não é suficientemente adequada para o sistema regional de gestão de recursos hídricos por ter leis, em sua maioria, escritas com base nos aspectos sociais e condições hidrológicas do sul do país.

Por sua vez, M.A.Oliveira; E.M.Barbosa; J. Dantas Neto (2013) colocam que a principal inovação da política hídrica nacional consiste na concepção de um modelo sistêmico de gestão, baseado nos princípios da descentralização, no planejamento compartilhado e na gestão participativa dos atores sociais.

\subsection{Gestão dos recursos}

Rebouças (1997), dando continuidade aos estudos de Campos e Vieira (1993), recusa o determinismo físico-climático que tem servido de justificativa à cultura da crise da água no Mundo ou no Brasil, bem como a cultura da seca na região Nordeste, afirmando que as condições físico-climáticas que predominam na região Nordeste do Brasil podem, relativamente, dificultar a vida, exigir maior empenho e maior racionalidade na gestão dos recursos naturais em geral e da água, em particular, no entanto não podem ser responsabilizadas pelo quadro de pobreza da região, justificando que o que mais falta não é água, mas determinado padrão cultural que agregue confiança e melhore a eficiência das organizações públicas e privadas envolvidas no negócio da água.

Tucci, Hespanhol e Netto (2000), corroborando com as ideias de Rebouças (1997), afirmam que a gestão de recursos hídricos passa por um cenário de transição institucional com a 
privatização dos serviços públicos e pela regulamentação da legislação de recursos hídricos e defendem que uma das prioridades nacionais do setor de recursos hídricos deve ser a preservação e aumento da disponibilidade de água em áreas críticas.

Em alternativa, Herman Bouwer (2000) propõe que mais armazenamento de água atrás de barragens e especialmente em aquíferos por meio de recarga artificial é necessário para economizar água em tempos de excedente para uso em períodos de escassez.

Ragab Ragab e Christel Prudhamme (2001), em concordância com Tucci, Hespanhol e Netto (2000), são favoráveis ao investimento em infraestrutura hídrica. Também apontam a construção de barragens para aumentar a eficiência com a qual as necessidades atuais, diante das mudanças climáticas, são atendidas e aumentar a eficiência com a qual a água é alocada entre diferentes usos em regiões áridas e semiáridas.

\subsection{Casos críticos}

No contexto nacional, Joaquim Gondim, Ana Paula Fioreze, Rodrigo Flecha Ferreira Alves e Wesley Gabrieli de Souza (2017) relacionam o quadro de seca do Semiárido Nordestino, a redução da disponibilidade hídrica e a manutenção dos usos da água, em especial, o abastecimento público, a irrigação e a geração hidrelétrica, ressaltando que os volumes dos reservatórios, essenciais para a segurança hídrica na região, vêm reduzindo significativamente, chegando ao colapso em muitos casos. Diante disso, sugerem uma rede robusta de sistemas de monitoramento e de instituições de gestão dos recursos hídricos para acompanhar, planejar e atuar com maior eficiência na redução dos efeitos da seca sobre a população.

\subsection{Abastecimento sustentável}

Hodiernamente, considerando o contexto da sociedade de consumo, Amador DuránSánchez; José Álvarez-García e María de la Cruz del Río-Rama (2018) definem a gestão sustentável dos recursos hídricos como uma prioridade essencial para atender à crescente demanda por água e alcançar um suprimento futuro seguro e ambientalmente sustentável. 


\section{Metodologia}

Foi realizado um estudo descritivo de ordem qualitativa e quantitativa, por meio de recorte temporal e regional. Para tanto, foram coletados dados de revistas e artigos, além de órgãos como ANA (Agência Nacional de Águas) e IBGE (Instituto Brasileiro de Geografia e Estatística).

Buscou-se dados relativos ao volume de água — em hectômetros cúbicos — disponível nos reservatórios da Bahia, Ceará, Maranhão, Paraíba, Pernambuco, Piauí e Rio Grande do Norte, no intervalo de dezembro de 2018 a dezembro de 2019. Vale ressaltar que foram utilizados somente dados dos estados com volumes armazenados iguais ou superiores a $10 \mathrm{hm}^{3}$, conforme critério adotado pela ANA. Posto isso, utilizou-se a ferramenta de giro de estoque para verificar as oscilações de abastecimento ao longo do período. Em seguida, utilizando o valor encontrado no giro e tomando como base o referido período, calculou-se o tempo em que o estoque de cada reservatório conseguiu atender às demandas sem necessidade de abastecimento (cobertura). Por fim, montou-se uma classificação ABC com o intuito de mostrar como os Estados pesquisados atendem às suas respectivas necessidades de abastecimento em termos de volume de água por habitante e por área.

A fim de possibilitar a execução dos demais cálculos, efetuou-se primeiramente o cálculo da capacidade equivalente de cada região, somando as capacidades dos reservatórios individualmente. Considerou-se o valor da capacidade constante. Em seguida, somou-se os volumes de todos os reservatórios individualmente conforme o período determinado (12/18 a 12/19) para então somar o valor de todos os meses e ter o volume equivalente. Por fim, dividiuse o volume equivalente pela capacidade equivalente para ter o valor do reservatório equivalente de cada região.

Para determinar o giro, fez-se a soma das saídas (consumo) dividida pelo EMMF (estoque médio mensal final), que é o resultado da soma dos estoques médios dividida pela quantidade de itens (meses). Para o cálculo de cobertura, dividiu-se o tempo - quantidade de meses vezes a quantidade de dias - pelo giro. A classificação ABC foi montada utilizando dados advindos da divisão do volume médio de água, transformado para $\mathrm{m}^{3}$, pelo número de habitantes de cada estado.

Ademais, ressalva-se que não foi levada em consideração para a realização dos cálculos a transposição das águas do rio São Francisco. 


\section{Resultados}

A Tabela 1 apresenta os dados mensais do volume em $\mathrm{hm}^{3}$ dos respectivos estados do nordeste além da soma equivalente dos volumes encontrados nos reservatórios de cada estado ao decorrer do ano.

Tabela 1 - Volume mensal dos reservatórios dos estados do nordeste (hm³) no ano de 2019.

\begin{tabular}{l|c|r|r|r|r|r|r|r}
\hline \multicolumn{1}{|c}{ Volume dos reservatórios do nordeste $\left(\mathrm{hm}^{3}\right)$} \\
\hline MÊS & \multicolumn{1}{|c|}{ Bahia } & \multicolumn{1}{c|}{ Ceará } & Paraiba & Pernambuco & Maranhão & \multicolumn{1}{c|}{ Piaui } & \multicolumn{1}{c|}{ RN } & Equivalente \\
\hline Janeiro & 1902,22 & 1849,78 & 652,12 & 492,23 & 730 & 758,72 & 925,51 & 7310,58 \\
\hline Fevereiro & 1827,64 & 1880,46 & 580,03 & 423,52 & 608,4 & 925,33 & 885,35 & 7130,73 \\
\hline Março & 1830,56 & 2190,66 & 538,92 & 399,01 & 608,4 & 925,33 & 901,66 & 7394,54 \\
\hline Abril & $1.213,91$ & $3.316,46$ & 743,13 & 531,87 & 608,4 & 925,33 & 1.258 & 8597,1 \\
\hline Maio & $1.185,34$ & $3.774,72$ & 756,92 & 518,42 & 659,1 & 925,33 & $1.352,93$ & 9172,76 \\
\hline Junho & $1.168,48$ & $3.849,76$ & 770,41 & 533,02 & 659,1 & 925,33 & $1.361,79$ & 9267,89 \\
\hline Julho & 1189,51 & 3826,67 & 805,2 & 593,37 & 659,1 & 1287,64 & 1357,35 & 9718,84 \\
\hline Agosto & 1156,17 & 3698,54 & 864,16 & 660,46 & 659,1 & 942,33 & 1311,77 & 9292,53 \\
\hline Setembro & 1130,29 & 3440,88 & 865,63 & 635,44 & 659,1 & 935,66 & 1247,35 & 8914,35 \\
\hline Outubro & 1780,53 & 3142,76 & 711,19 & 525,81 & 660 & 840,95 & 1132,92 & 8794,16 \\
\hline Novembro & 1719,23 & 2916,46 & 652,36 & 482,07 & 660 & 838,01 & 1067,92 & 8336,05 \\
\hline Dezembro & 1654,24 & 1988,84 & 628,2 & 488,95 & 660 & 759,72 & 963,82 & 7143,77 \\
\hline Cap. Total: & $4.352,76$ & $18.654,65$ & 1014 & 8705,77 & 4371,02 & 3046,19 & 1682,55 & $41.826,94$ \\
\hline
\end{tabular}

Fonte: Adaptado da ANA

Obtendo-se a soma total das capacidades dos reservatórios, considerando apenas os maiores ou iguais a $10 \mathrm{hm}^{3}$, têm-se o valor de $41.826,94 \mathrm{hm}^{3}$. Comparando a capacidade total com a soma dos volumes de cada estado, verifica-se que: no mês de janeiro/2019, o volume total corresponde a apenas $17,45 \%$ da capacidade total dos reservatórios, enquanto no mês subsequente, fevereiro/2019, o volume baixa para $17 \%$, março $17,67 \%$, abril $20,55 \%$, maio $21,93 \%$, junho $22,15 \%$, julho $23,23 \%$ (sendo o maior volume ao decorrer do ano), agosto $22,21 \%$, setembro $21,31 \%$, outubro $21 \%$, novembro $19,92 \%$ e dezembro $17 \%$ (sendo o menor volume ao decorrer do ano).

A Tabela 2 foi criada a fim de viabilizar o cálculo de giro. Nela estão dispostas as categorias início, entrada, saída, estoque final e estoque médio. 
Tabela 2 - Entrada e saída do volume de água nos reservatórios equivalentes do Nordeste (hm³)

\begin{tabular}{l|r|r|r|r|r}
\multicolumn{1}{c|}{ Mês } & Inicio & Entrada & Saida & Estoque Final & Estoque Médio. \\
\hline Janeiro & $7.151,70$ & 158,88 & 0 & $7.310,58$ & 7231,14 \\
\hline Fevereiro & $7.310,58$ & 0 & 179,85 & $7.130,73$ & 7220,655 \\
\hline Março & $7.130,73$ & 263,81 & 0 & $7.394,54$ & 7262,635 \\
\hline Abril & $7.394,54$ & $1.202,56$ & 0 & $8.597,10$ & 7995,82 \\
\hline Maio & 8597,1 & 575,66 & 0 & 9172,76 & 8884,93 \\
\hline Junho & 9172,76 & 95,13 & 0 & 9267,89 & 9220,325 \\
\hline Julho & 9267,89 & 450,95 & 0 & 9718,84 & 9493,365 \\
\hline Agosto & 9718,84 & 0 & 426,31 & 9292,53 & 9505,685 \\
\hline Setembro & 9292,53 & 0 & 378,18 & 8914,35 & 9103,44 \\
\hline Outubro & 8914,35 & 0 & 120,19 & 8794,16 & 8854,255 \\
\hline Novembro & 8794,16 & 0 & 458,11 & 8336,05 & 8565,105 \\
\hline Dezembro & 8336,05 & 0 & 1192,28 & 7143,77 & 7739,91 \\
\hline SOMA T. & & & 2754,92 & $101.073,30$ & 101077,265 \\
\hline
\end{tabular}

Fonte: Adaptado da ANA

O reservatório equivalente de dezembro de 2018 foi adotado como valor inicial; foi considerado que houve uma entrada quando verificado que o reservatório equivalente aumentou de um mês para o outro e saída quando diminuiu, sendo a entrada ou saída essa diferença. Já o estoque final é a soma dos inícios mais as entradas subtraindo-se as saídas, enquanto o estoque médio é a soma dos valores iniciais e os estoques finais divididos por dois. Nota-se que um dos valores, de entrada ou saída, é sempre 0 , o que, é importante salientar, não corresponde a ausência de chuvas ou de consumo, mas se trata de um arredondamento, no qual o valor 0 significa que o valor acrescido se equivale ao consumido de modo a ser desprezível.

A Tabela 3 descreve os procedimentos que foram realizados com os dados obtidos sobre os reservatórios para calcular o estoque médio mensal final e o giro.

Tabela 3. Cálculo do estoque médio mensal, giro e cobertura.

\begin{tabular}{l|r|l|r|r|r}
\multicolumn{2}{c|}{ Estoque médio mensal final } & \multicolumn{2}{c}{ COBERTURA } \\
\hline Soma EM. $\left(\mathrm{Hm}^{3}\right)$ & 101077,265 & EMMF: & 8423,1 & Tempo (dias) & 360 \\
\hline Qtd. Itens & 12 & Soma. Saídas & 2754,92 & Giro & 0,32 \\
\hline EMMF: & 8423,11 & GIRO: & 0,327067232 & Cobertura & 1125 \\
\hline
\end{tabular}

Fonte: Adaptado da ANA

Para calcular o estoque médio mensal final (EMMF) realizou-se a soma dos estoques médios de cada mês e dividiu-se o valor encontrado pelo total de meses. O resultado obtido é 
o volume no qual os reservatórios se mantêm. Ao dividir o EMMF pela somatória de todas as saídas encontra-se giro de aproximadamente 0,32 ou $32 \%$, o que significa que não ocorre uma renovação de recursos considerável, ou seja, ocorrem poucas chuvas e a água permanece "parada" nos reservatórios por longos períodos. Com essa informação, calcula-se a cobertura dividindo o giro pelo tempo contado em dia (12 meses=360 dias) e verifica-se que é possível permanecer com o mesmo estoque por em torno de três anos e um mês (1125 dias). Todavia, se o giro se alterar com o tempo, seja pelo EMMF ou soma das saídas, essa realidade pode mudar.

A tabela 4 indica o valor mensal de cada estado, da razão entre o volume $\left(\mathrm{em} \mathrm{m}^{3}\right)$ presentes nos reservatórios pelo devido valor populacional de cada estado. Sendo feita uma média anual desses valores a fim da execução de uma classificação $\mathrm{ABC}$, ferramenta essa, utilizada pela logística para um melhor desempenho na gestão.

Tabela 4. Relação entre volume $\left(\mathrm{m}^{3}\right)$ e população.

\begin{tabular}{l|r|r|r|r|r|r|r} 
& \multicolumn{1}{|c|}{ Bahia } & \multicolumn{1}{c|}{ Ceará } & Paraiba & Pernambuco & Maranhão & \multicolumn{1}{c}{ Piauí } & \multicolumn{1}{c}{ RN } \\
\hline Janeiro & 127,60 & 201,82 & 161,83 & 51,31 & 102,83 & 231,50 & 262,65 \\
\hline Fevereiro & 122,60 & 205,17 & 143,94 & 44,14 & 85,70 & 282,34 & 251,25 \\
\hline Março & 122,80 & 239,02 & 133,74 & 41,59 & 85,70 & 282,34 & 255,88 \\
\hline Abril & 81,43 & 361,85 & 184,41 & 55,44 & 85,70 & 282,34 & 357,00 \\
\hline Maio & 79,51 & 411,85 & 187,84 & 54,04 & 92,84 & 282,34 & 383,94 \\
\hline Junho & 78,38 & 420,04 & 191,18 & 55,56 & 92,84 & 282,34 & 386,45 \\
\hline Julho & 79,79 & 417,52 & 199,82 & 61,85 & 92,84 & 392,88 & 385,19 \\
\hline Agosto & 77,56 & 403,54 & 214,45 & 68,84 & 92,84 & 287,52 & 372,26 \\
\hline Setembro & 75,82 & 375,43 & 214,81 & 66,23 & 92,84 & 285,49 & 353,98 \\
\hline Outubro & 119,44 & 342,90 & 176,49 & 54,81 & 92,97 & 256,59 & 321,51 \\
\hline Novembro & 115,33 & 318,21 & 161,89 & 50,25 & 92,97 & 255,69 & 303,06 \\
\hline Dezembro & 110,97 & 217,00 & 155,89 & 50,96 & 92,97 & 231,81 & 273,52 \\
\hline Média: & 99,27 & 326,19 & 177,19 & 54,58 & 91,92 & 279,43 & 325,56 \\
\hline
\end{tabular}

Fonte: Adaptado da ANA

A Tabela 5 mostra a construção de uma classificação ABC a fim de classificar a relação entre o volume presente em cada estado e a quantidade de pessoas. 
Tabela 5. Classificação ABC da razão entre o volume e a população estadual.

\begin{tabular}{c|r|r|r|c} 
Estado & Volume/População & P.I & P.A & Classificação \\
\hline Ceará & 326,19 & $24 \%$ & $24 \%$ & A \\
\hline RN & 325,56 & $24 \%$ & $48 \%$ & A \\
\hline Piauí & 279,43 & $21 \%$ & $69 \%$ & B \\
\hline Paraiba & 177,19 & $13 \%$ & $82 \%$ & B \\
\hline Bahia & 99,27 & $7 \%$ & $89 \%$ & C \\
\hline Maranhão & 91,92 & $7 \%$ & $96 \%$ & C \\
\hline Pernambuco & 54,58 & $4 \%$ & $100 \%$ & C \\
\hline Soma total: & 1354,14 & & &
\end{tabular}

Fonte: Adaptado do IBGE

Estão dispostas as categorias: estado, consumo, porcentagem individual e porcentagem acumulada. O consumo foi dado a partir da média da razão entre o volume presente nos reservatórios e o número de pessoas nos respectivos estados, sendo organizados de ordem decrescente para a execução da ferramenta. Foi então realizada a soma total das médias a fim de identificar a porcentagem individual de cada estado, logo após, com os valores encontrados foi possível encontrar as porcentagens acumuladas de cada estado. E por fim, foi realizada a classificação $\mathrm{ABC}$, que por sua vez, mostra os estados que possuem uma boa quantidade de água para suprir as necessidades de sua população, sendo classificados como "A". E indica os estados que requerem uma atenção maior quanto a distribuição de água para a sua população, sendo eles classificados como " $\mathrm{C}$ ".

Na Tabela 6 estão indicadas a razão entre valor mensal dos volumes dos reservatórios $\left(\mathrm{em} \mathrm{m}^{3}\right)$ e área $\left(\mathrm{em} \mathrm{Km}^{2}\right)$ dos respectivos estados. Além da média anual dos valores de cada estado para a utilização da classificação ABC. 
Tabela 6 - Relação entre Volume $\left(\mathrm{m}^{3}\right)$ pela área $\left(\mathrm{Km}^{2}\right)$.

\begin{tabular}{l|r|r|r|r|r|r|r} 
& Bahia & \multicolumn{1}{c|}{ Ceará } & \multicolumn{1}{c|}{ Paraiba } & Pernambuco & \multicolumn{1}{c}{ Maranhão } & \multicolumn{1}{c}{ Piaúi } & \multicolumn{1}{c}{ RN } \\
\hline Janeiro & 3353,141 & 12429,145 & 11524,609 & 521,862 & 309,740 & 920,374 & 17529,594 \\
\hline Fevereiro & 3221,675 & 12635,292 & 10250,596 & 449,016 & 258,145 & 1122,482 & 16768,945 \\
\hline Março & 3226,822 & 14719,605 & 9524,079 & 423,030 & 258,145 & 1122,482 & 17077,864 \\
\hline Abril & 2139,821 & 22284,144 & 13132,986 & 563,888 & 258,145 & 1122,482 & 23827,111 \\
\hline Maio & 2089,460 & 25363,310 & 13376,690 & 549,629 & 279,657 & 1122,482 & 25625,130 \\
\hline Junho & 2059,740 & 25867,523 & 13615,092 & 565,107 & 279,657 & 1122,482 & 25792,943 \\
\hline Julho & 2096,810 & 25712,376 & 14229,920 & 629,091 & 279,657 & 1561,986 & 25708,847 \\
\hline Agosto & 2038,040 & 24851,437 & 15271,892 & 700,219 & 279,657 & 1143,104 & 24845,540 \\
\hline Setembro & 1992,420 & 23120,154 & 15297,870 & 673,693 & 279,657 & 1135,013 & 23625,395 \\
\hline Outubro & 3138,632 & 21117,009 & 12568,525 & 557,463 & 280,039 & 1020,124 & 21458,037 \\
\hline Novembro & 3030,575 & 19596,441 & 11528,850 & 511,090 & 280,039 & 1016,558 & 20226,907 \\
\hline Dezembro & 2916,014 & 13363,525 & 11101,882 & 518,384 & 280,039 & 921,587 & 18255,204 \\
\hline Média: & 2608,596 & 20088,330 & 12618,583 & 555,206 & 276,882 & 1110,930 & 21728,460 \\
\hline
\end{tabular}

Fonte: Adaptado da ANA

A Tabela 7 foi montada com o intuito de classificar os estados a partir da razão entre o volume presente nos reservatórios e a área dos respectivos estados.

Tabela 7. Classificação ABC do volume por $\mathrm{km}^{2}$

\begin{tabular}{c|r|r|r|c} 
Estado & \multicolumn{1}{|c|}{ Consumo } & \multicolumn{1}{c|}{ P.I } & \multicolumn{1}{c|}{ P.A } & Classificação \\
\hline RN & 21728,46 & $37 \%$ & $37 \%$ & A \\
\hline Ceará & 20088,33 & $34 \%$ & $71 \%$ & B \\
\hline Paraiba & 12618,583 & $21 \%$ & $92 \%$ & C \\
\hline Bahia & 2608,596 & $4 \%$ & $97 \%$ & C \\
\hline Piauí & 1110,93 & $2 \%$ & $99 \%$ & C \\
\hline Pernambuco & 555,206 & $1 \%$ & $100 \%$ & $\mathrm{C}$ \\
\hline Maranhão & 276,882 & $0 \%$ & $100 \%$ & $\mathrm{C}$ \\
\hline Soma total: & 58986,987 & & &
\end{tabular}

Fonte: Adaptado da ANA

Estão expostas as categorias: estado, volume/área, porcentagem individual e porcentagem acumulada. Foi realizada a soma total das médias a fim de identificar a porcentagem individual de cada estado, logo após, com os valores encontrados, foi possível definir as porcentagens acumuladas de cada estado. Os estados foram classificados de acordo com a quantidade de água $\mathrm{em}^{3}$ por $\mathrm{km}^{2}$. Sendo assim, a tabela classifica os estados que possuem uma grande quantidade de volume por $\mathrm{km}^{2}$ como "A", e classifica os estados que apesar de uma escala territorial considerável, possuem um baixo volume por $\mathrm{km}^{2}$, como " $\mathrm{C}$ ". 


\section{Considerações finais}

Esse trabalho teve como objetivo analisar a atual situação dos reservatórios hidrográficos da região Nordeste sob a ótica da implementação do uso de ferramentas de gestão logística com a finalidade de otimizar o planejamento dos recursos hídricos da região.

A partir de uma pesquisa de campo seguida da aplicação demonstrativa de algumas das ferramentas fundamentais da área da gestão logística foi possível obter dados relevantes de giro, cobertura, consumo e alcance populacional e territorial do volume de água armazenado nos reservatórios estudados, alcançando assim os objetivos propostos.

Essa pesquisa se fez necessária haja visto que os atuais métodos de planejamento utilizados, embora parcialmente eficazes, não se fazem suficientes para atender integralmente às demandas de consumo, de modo que a crise hídrica do Nordeste está sempre em pauta, requerendo investimentos em estudos e novas abordagens de tratamento para esse quadro.

Nesse sentido, a integração de áreas do conhecimento com a logística para futuras pesquisas pode ser um diferencial na gestão dos recursos hídricos, partindo do pressuposto de que toda cadeia de produção ou de suprimento, por mais indiretamente que possa se apresentar, deve ser analisada de um ponto de vista estratégico para reduzir perdas e otimizar os ganhos. Espera-se, portanto, incentivar com esse trabalho a adoção do uso de ferramentas logísticas como subsídio para um melhor planejamento hídrico por parte dos Estados do Nordeste.

\section{REFERÊNCIAS}

ANA. Boletim de acompanhamento dos reservatórios do nordeste do Brasil. Disponível em: file:///C:/Users/20181161210026/Downloads/02-

Boletim_Monitoramento_Reser_Nordeste_15\%20de\%20Janeiro.pdf. Acesso em: 20 jan. 2020.

ASSOULINE, S.; NARKIS, K.; OR, D. Evaporation suppression from water reservoirs: Efficiency considerations of partial covers. Disponível em: https://agupubs.onlinelibrary.wiley.com/doi/pdf/10.1029/2010WR009889. Acesso em: 11 dez. 2019.

ÁLVAREZ-GARCIA, J.; CRUZ, M. R. R.; DURÁN-SÁNCHEZ, A. Sustainable Water Resources Management: A Bibliometric Overview. Disponível em: https://www.mdpi.com/2073-4441/10/9/1191/pdf. Acesso em: 22 out. 2019.

BARRETO, B. F. F.; FREITAS, D. L.; QUEIROZ, R. D.; VALENTE, L. S. O nordeste e a escassez de água: uma abordagem da microrregião de Pau dos Ferros/RN. Disponível em: 
http://anpur.org.br/xviienanpur/principal/publicacoes/XVII.ENANPUR_Anais/ST_Sessoes_Tematicas/ST\%204/ ST\%204.6/ST\%204.6-03.pdf. Acesso em: 22 out. 2019.

BOUWER, H. Integrated Water management: emerging issues and challenges. Disponível em:http://citeseerx.ist.psu.edu/viewdoc/download?doi=10.1.1.476.6599\&rep=rep1\&type=pdf. Acesso em: 22 out. 2019.

BESERRA, J. N. C.; VIEIRA, V. P. P. B. Gerenciamento dos recursos hídricos: A problemática do nordeste. Disponível em: http://bibliotecadigital.fgv.br/ojs/index.php/rap/article/viewFile/8669/7400. Acesso em: 22 out. 2019.

BARBOSA, E.M.; DANTAS NETOS, J. OLIVEIRA, M. A.;Gestão de recursos hídricos no Rio Grande do Norte: Uma análise da implementação da política hídrica. Disponível em: http://www2.ifrn.edu.br/ojs/index.php/HOLOS/article/viewFile/1186/634. Acesso em: 6 nov. 2019.

CORDEIRO, O. M. N.; HESPENHOL, I.; TUCCL, C. E. M. Cenários da gestão da água no Brasil: Uma contribuição para a “visão mundial da água”. Disponível em: http://rhama.com.br/blog/wpcontent/uploads/2017/01/cenarios-da-gestao-da-agua-no-brasil-1.pdf. Acesso em: 6 out. 2019.

CABRAL, T. S. et al. Planejamento dos recursos hídricos na bacia hidrográfica do Rio Gramame, uma bacia Litorânea do Nordeste Brasileiro. Disponível em: https://abrh.s3.sa-east1.amazonaws.com/Sumarios/101/3e0d7dbbd001a2b1573f8a9101ad2979_2f9a45d5932a52ea71cd1fb07b40e6d4 .pdf. Acesso em: 7 out. 2019.

FADUL, E.; MENDONÇA, J.L.M.; SANTOS, L. C. Limites e desafios na gestão de recursos hídricos por comitês de Bacias hidrográficas: Um estudo nos estados do nordeste do Brasil. Disponível em: http://www.scielo.br/pdf/read/v24n1/1413-2311-read-24-1-238.pdf. Acesso em: 10 dez. 2019.

GONDIM, J. et al. A seca atual no Semiárido nordestino - Impactos sobre os recursos hídricos. Disponível em: http://seer.cgee.org.br/index.php/parcerias_estrategicas/article/viewFile/856/784. Acesso em 10 dez. 2019

LA LAINA, R. P.; PORTO, M. F. A. Gestão de Bacias Hidrográficas. Disponível em: http://www.scielo.br/pdf/ea/v22n63/v22n63a04.pdf. Acesso em: 30 out. 2019.

OLIVEIRA, P. T. S. et al. Caracterização morfométrica de bacias hidrográficas através de dados SRTM. Disponível em: https://repositorio.ufms.br:8443/jspui/bitstream/123456789/731/1/Caracterizacao\%20morfometrica.pdf. Acesso em: 15 jan. 2019.

PRUDHAME, C.; RAGAB, R. Climate Change and Water Resources Management in Arid and Semi-arid Regions: Prospective and Challenges for the 21st Century. Disponível em: https://pdfs.semanticscholar.org/f7b3/267a57191ff3238da7d8960d253680f3503e.pdf. Acesso em: 22. Jan 2019. 
REBOUÇAS, A. C. Água na região Nordeste: Desperdício e escassez. Disponível em: http://www.scielo.br/pdf/ea/v11n29/v11n29a07.pdf. Acesso em: 12 dez. 2019. 